\title{
Bottom-Up Fabrication of Sulfur-Doped Graphene Films De- rived from Sulfur-Annulated Nanographene for Ultrahigh Vol- umetric Capacitance Micro-Supercapacitors
}

\author{
Zhong-Shuai Wu, ${ }^{, \dagger}$ Yun-Zhi Tan, ${ }^{\ddagger}$ Shuanghao Zheng, ${ }^{\dagger, \xi_{*}}$ Sen Wang, ${ }^{\dagger}{ }^{*}$ Khaled Parvez, Jieqiong Qin, ${ }^{\dagger}$, \\ Xiaoyu Shi, ${ }^{\dagger}, \S$ Chenglin Sun, ${ }^{\dagger}, "$ Xinhe Bao, ${ }^{\dagger, \S}$ Xinliang Feng, ${ }^{*}$, and Klaus Müllen, ${ }^{*, *}$ \\ †Dalian National Laboratory for Clean Energy, Dalian Institute of Chemical Physics, Chinese Academy of Sciences \\ 457 Zhongshan Road, Dalian 116023, China \\ "State Key Laboratory for Physical Chemistry of Solid Surfaces and Department of Chemistry College of Chemistry \\ and Chemical Engineering, Xiamen University, 422 Siming South Road, Xiamen, 361005, China \\ \$State Key Laboratory of Catalysis, Dalian Institute of Chemical Physics, Chinese Academy of Sciences, 457 \\ Zhongshan Road, Dalian 116023, China \\ University of Chinese Academy of Sciences, 19 A Yuquan Rd, Shijingshan District, Beijing, 100049, China \\ 'School of Chemistry, University of Manchester, Oxford Road, Manchester M13 9PL, UK \\ 'Center for Advancing Electronics Dresden (cfaed) \& Department of Chemistry and Food Chemistry, Technische \\ Universität Dresden, Mommsenstraße 4, 01062 Dresden, Germany \\ ${ }^{\ddagger}$ Max-Planck-Institut für Polymerforschung, Ackermannweg 10, 55128 Mainz, Germany \\ Supporting Information Placeholder
}

\begin{abstract}
Heteroatom doping of nanocarbon films can efficiently boost the pseudocapacitance of microsupercapacitors (MSCs), however, wafer-scale fabrication of sulfur-doped graphene films with a tailored thickness and homogeneous doping for MSCs remains a great challenge. Here we demonstrate the bottom-up fabrication of continuous, uniform, ultrathin sulfur-doped graphene (SG) films, derived from the peripherical tri-sulfur-annulated hexa-perihexabenzocoronene (SHBC), for ultrahigh-rate MSCs (SG-MSCs) with landmark volumetric capacitance. The SG film was prepared by thermal annealing of the spray-coated SHBC-based film, with assistance of a thin Au protecting layer, at 800 ${ }^{\circ} \mathrm{C}$ for $30 \mathrm{~min}$. SHBC with twelve phenylthio groups decorated at the periphery is critical as precursor for the formation of the continuous and ultrathin SG film, with a uniform thickness of $\sim 10.0 \mathrm{~nm}$. Notably, the as-produced all-solid-state planar SG-MSCs exhibited a highly stable pseudocapacitive behavior with an volumetric capacitance of $\sim 582 \mathrm{~F} \mathrm{~cm}^{-3}$ at $10 \mathrm{mV}$ $\mathrm{s}^{-1}$, excellent rate capability with a remarkable capacitance of $8.1 \mathrm{~F} \mathrm{~cm}^{-3}$ even at an ultrahigh rate of $2000 \mathrm{~V} \mathrm{~s}^{-1}$, ultrafast frequency response with a short time constant of $0.26 \mathrm{~ms}$, and ultrahigh power density of $\sim 1191 \mathrm{~W} \mathrm{~cm}^{-3}$. It is noteworthy that these values obtained are among the best values for carbon-based MSCs reported to date.
\end{abstract}

\section{INTRODUCTION}

The current development of flexible and wearable electronics concentrates on the continuous miniaturization and diversified integration of micro-scale power sources. ${ }^{1-4}$ The conventional energy storage devices such as lithium ion batteries and supercapacitors, however, are usually configured with sandwich-like stacked geometries which poorly miniaturize in size and are not well compatible with planar integrated circuits on one single substrate. ${ }^{5-7}$ In this regard, the emerging planar micro-supercapacitor (MSC) device components, including two electrodes, separator, electrolyte, and current collectors of MSCs are readily constructed on one single substrate. Furthermore, the MSCs possess desirable merits of ultrahigh power delivery, outstanding rate capability, and high-frequency response. ${ }^{8-10}$ Notable efforts have been devoted to the development of advanced thin-film electrode materials of nanostructured carbon materials, metal oxides, and conducting polymers for MSCs. ${ }^{11,12}$ The available metal oxides, e.g., $\mathrm{RuO}_{2},{ }^{13} \mathrm{MnO}_{2},{ }^{14} \mathrm{Ni}(\mathrm{OH})_{2},{ }^{15} \mathrm{CoO},{ }^{16}$ and conducting polymers, e.g., polyaniline, ${ }^{17}$ polypyrrole, ${ }^{18}$ generally show high volumetric capacitance of $300 \sim 800 \mathrm{~F} \mathrm{~cm}^{-3}$, but suffer from limited power delivery, slow frequency response and short cycling stability because of poor electrode kinetics. ${ }^{19,20}$ As a consequence, "nanocarbons", including activated carbon $(\mathrm{AC}),{ }^{21,22}$ carbide-derived carbon (CDC), onion-like carbon (OLC), ${ }^{23}$ carbon nanotubes (CNTs), ${ }^{24}$ and graphene ${ }^{25-30}$ appear as most promising candidates for MSCs. 


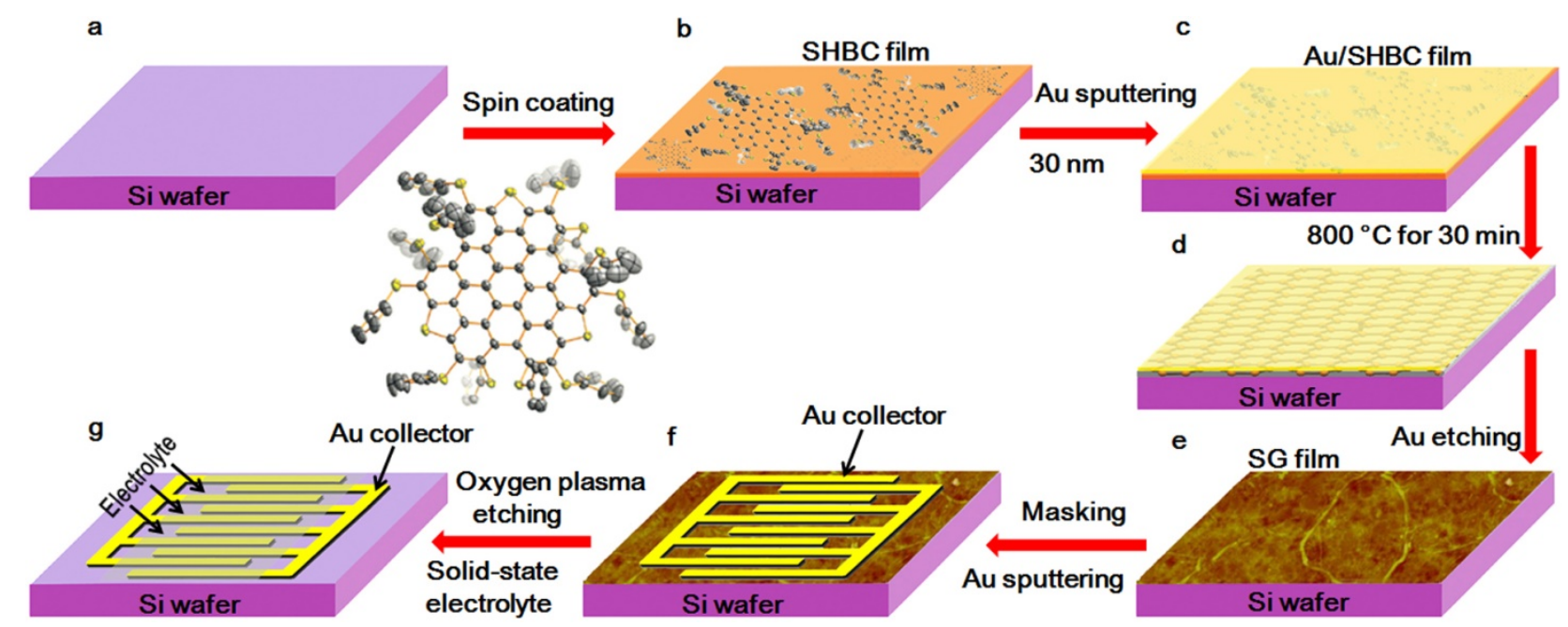

Figure 1. Schematic illustration of SHBC-derived SG films for planar MSCs on a $\mathrm{Si} / \mathrm{SiO}_{2}$ wafer. (a) Spin-coating of the SHBC solution on surface-modified silicon with oxygen plasma treatment. (b) Sputtering Au with a thickness of $30 \mathrm{~nm}$ on the SHBC film. (c) Thermal annealing at $800{ }^{\circ} \mathrm{C}$ for $30 \mathrm{~min}$. (d) Au etching by a $\mathrm{KI} / \mathrm{I}_{2}$ aqueous solution. (e) Masking micropatterns and deposition of gold current collector. (f) Oxygen plasma etching and drop casting of $\mathrm{H}_{2} \mathrm{SO}_{4} / \mathrm{PVA}$ gel electrolyte on interdigitated fingers. (g) All-solid-state SG-MSCs obtained after solidification of gel electrolyte.

The incorporation of single or dual heteroatoms (e.g., $\mathrm{N}^{31-}$ ${ }^{33} \mathrm{~B},{ }^{34-36} \mathrm{P},{ }^{37-39} \mathrm{~S},{ }^{40-42} \mathrm{O}^{43}$ ) into the nanocarbons is a practical strategy to considerably enhance the capacitance through additional Faradaic reactions generally called pseudocapacitance effects. ${ }^{44,45}$ Special emphasis is given to sulfur doping. Sulfur has a similar electronegativity (2.58) as carbon (2.55) in the graphitic layers, ${ }^{46}$ which is also effective in modifying the electronic arrangement of graphitic lattice, as doped by larger electronegative nitrogen (3.04). ${ }^{47}$ Recent theoretical and experimental studies have proven that the presence of sulfur heteroatoms could substantially modify spin densities on graphene, ${ }^{47,48}$ producing significantly improved materials for supercapacitors. ${ }^{40,41}$ In particular, C-S bonds at the edge or near defects are important active sites, which greatly influence the charging of the electrical double layer and eventually facilitate the pseudocapacitance behavior. $^{42}$ In addition, bottom-up synthesis is a reliable strategy towards high yielding production of defect-free molecular graphene $(\leq 5 \mathrm{~nm})$, nanographene $(5-500 \mathrm{~nm})$ and macrographene $(\geq 500 \mathrm{~nm})$ through controlled chemical reaction or thermolysis of structurally-defined precursors, e.g., polycyclic aromatic hydrocarbons. ${ }^{49}$ With this approach, the structure of the precursor together with chemical or thermal reactions will determine the structure of the product, which is critical for the growth and integration of structurally-defined large graphene and graphene-based materials, ${ }^{49}$ showing exciting properties for electronic, optoelectronic and energy devices. ${ }^{50}$ Although planar MSCs based on nitrogen ${ }^{51}$ and boron $^{52}$ doped carbon films have been reported, bottom-up fabrication of thin sulfur-doped carbon films with a tailored thickness and homogeneous doping for MSCs has remained elusive.

Herein we develop the bottom-up fabrication of continuous, uniform, ultrathin sulfur-doped graphene (SG) films, derived from the peripherical tri-sulfur-annulated hexa-perihexabenzocoronene (SHBC, nanographene), for ultrahigh volumetric capacitance MSCs (denoted as SG-MSCs). The wafer-scale SG film with a uniform thickness of $\sim 10.0 \mathrm{~nm}$ was produced by thermal annealing of the spray-coated SHBC- based film under a thin Au protecting layer, at $800{ }^{\circ} \mathrm{C}$ for 30 min. Remarkably, the as-produced all-solid-state planar SGMSCs presented outstanding pseudocapacitive behavior with unprecedented volumetric capacitance of $\sim 582 \mathrm{~F} \mathrm{~cm}^{-3}(10 \mathrm{mV}$ $\mathrm{s}^{-1}$ ), excellent rate capability with a remarkable capacitance of 8.1 $\mathrm{F} \mathrm{cm}^{-3}$ even at an ultrahigh rate of $2000 \mathrm{~V} \mathrm{~s}^{-1}$, an extremely short time constant of $0.26 \mathrm{~ms}$, and ultrahigh power density of $\sim 1191 \mathrm{~W} \mathrm{~cm}^{-3}$. It is highlighted that these values obtained are among the best values for carbon-based MSCs reported to date.

\section{RESULTS AND DISCUSSION}

Fabrication of SG-MSCs. Figure 1 schematically depicts the stepwise fabrication of the planar SG-MSCs on a $\mathrm{Si} / \mathrm{SiO}_{2}$ wafer. First, the SHBC with twelve phenylthio groups decorated at the periphery was synthesized by thiolation of perchlorinated HBC (Figure $\mathrm{S}_{1}$ in Supporting Information), as described in our previous work. ${ }^{53,54}$ Then, a thin SHBC film was obtained by spin-coating a SHBC dispersion $\left(0.5 \mathrm{mg} \mathrm{mL}^{-1}\right.$, Figure S2a) on an oxygen plasma treated silicon wafer (Figure $1 \mathrm{a}, \mathrm{b})$. After sputtering a thin Au layer with a thickness of $30 \mathrm{~nm}$ (Figure 1c, Figure S2b), the SHBC film was thermally treated at $400{ }^{\circ} \mathrm{C}$ for $30 \mathrm{~min}$ (denoted as $\mathrm{SHBC}_{400}$ ), and then at $800{ }^{\circ} \mathrm{C}$ for $30 \mathrm{~min}$ (Figure 1d). It should be mentioned that the surface coverage of thin Au layer is helpful for the adsorption of cleaved sulfurs of twelve thiol groups of $\mathrm{HBC}$ molecule on the gold surface via the S-Au bonds, ${ }^{55}$ and simultaneous reorganization of these S-terminated HBC molecules via the cleavage of C-S bonds in phenylthio groups to form a surface-attached film. Further, the Au layer can be regarded as the two-dimensional $(2 \mathrm{D})$ catalytic cover, ${ }^{56,57}$ which provides a $2 \mathrm{D}$ confinement effect for the continuous growth of SHBC-derived SG film during annealing process. As expected, a wafer-scale film could not be obtained without a confining Au layer and without thermal annealing at $400{ }^{\circ} \mathrm{C}$ (Figure $\mathrm{S}_{3}$ ), or when replacing the precursor SHBC by pristine HBC (hexa-tert-butyl-hexa-perihexabenzocoronene), without thiol groups decorated at the periphery (Figure $\mathrm{S}_{4}$ ). After removing the Au layer by etching 

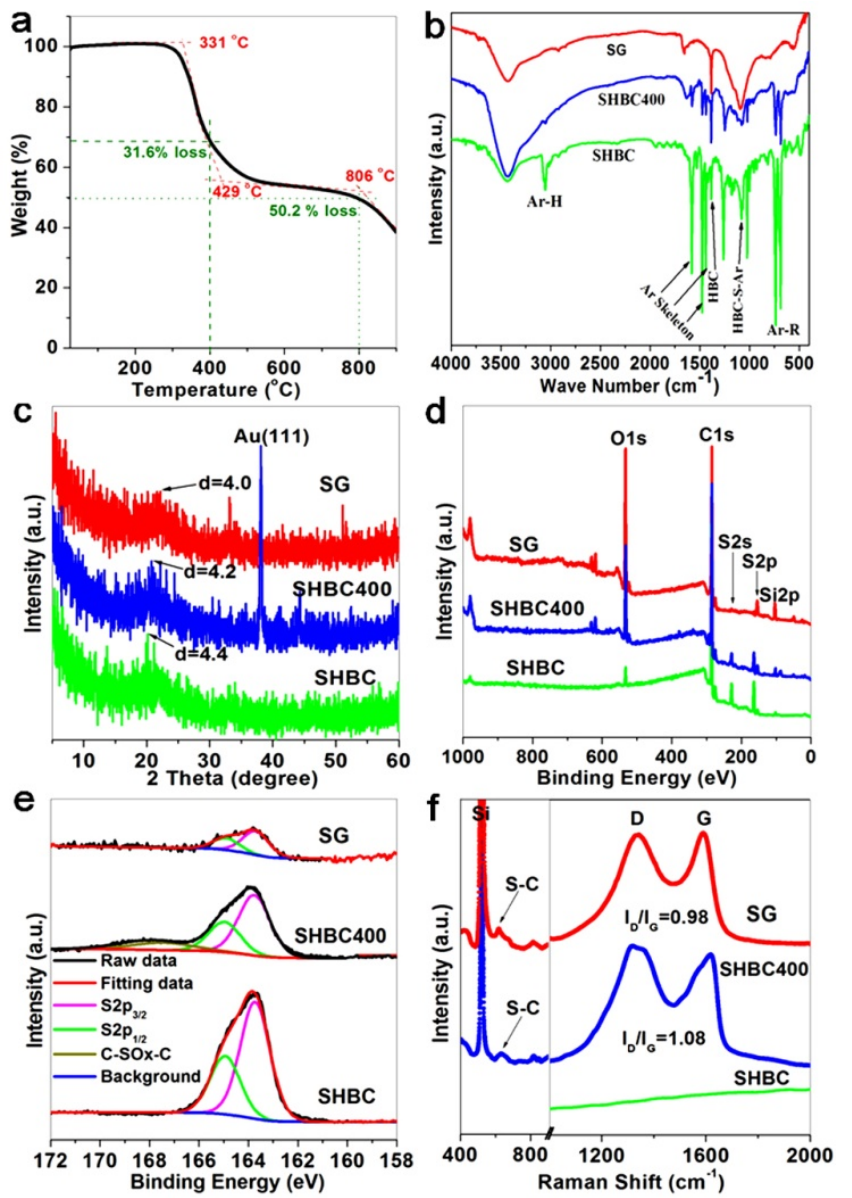

Figure 2. Structural characterization of SG, $\mathrm{SHBC}_{400}$ and SHBC films. (a) TGA curve of SHBC measured from 25 to 900 ${ }^{\circ} \mathrm{C}$ in nitrogen atmosphere with a heating rate of $5{ }^{\circ} \mathrm{C} / \mathrm{min}$. (b) FT-IR spectra (Ar stands for aromatic core or benzene ring), (c) XRD patterns, (d) overview XPS spectra, (e) highresolution S2p XPS spectra, and (f) Raman spectra of SG, $\mathrm{SHBC}_{400}$ and SHBC films.

with a $\mathrm{KI} / \mathrm{I}_{2}$ solution (Figure 1e, Figure S2c), the SG-based interdigitated microelectrode patterns were created through the deposition of gold current collectors, followed by oxidative etching in an oxygen plasma (Figure if,g). Finally, a polymer gel electrolyte of $\mathrm{H}_{2} \mathrm{SO}_{4}$ /polyvinyl alcohol $\left(\mathrm{H}_{2} \mathrm{SO}_{4} / \mathrm{PVA}\right)$ was drop-cast onto the interdigitated fingers, and the planar SG-MSCs were obtained after solidification overnight (Figure ig).

Characterization of SG Film. The structural evolution of SG films, SHBC40o and SHBC, was evaluated based on thermogravimetric analysis (TGA), Fourier transform infrared (FT-IR) spectroscopy, X-ray diffraction (XRD) patterns, X-ray photoelectron spectroscopy (XPS) and Raman spectra (Figure 2). In TGA curve (Figure 2a), SHBC showed a major mass reduction of approximately $50.2 \mathrm{wt} \%$ up to $800{ }^{\circ} \mathrm{C}$, which was basically close to the theoretical weight loss $\sim 50.3$ $w t \%$ of twelve phenyl groups. This is suggested the cleavage of C-S bonds in phenylthio groups. Note that the aromatic $\mathrm{HBC}$ units were thermally stable until $80{ }^{\circ} \mathrm{C}$, in accordance with the literature. ${ }^{5^{8}}$ The loss of the phenyl groups from SHBC at $800{ }^{\circ} \mathrm{C}$ was further identified by FT-IR (Figure $2 \mathrm{~b}$ ). SHBC exhibited characteristic bands, including $3046 \mathrm{~cm}^{-1}$ for the $\mathrm{C}-\mathrm{H}$ stretching vibration, ${ }^{58} 1580,1527,1476$ and $1437 \mathrm{~cm}^{-1}$ for benzene ring stretching vibrations, $686 \mathrm{~cm}^{-1}$ for the $\mathrm{C}-\mathrm{H}$ deformation vibration of the phenyl groups, ${ }^{58}$ and $736 \mathrm{~cm}^{-1}$ for out-of-plane bending mode of the aromatic core, ${ }^{57}$ respectively. However, with increased temperature, it was observed that the total intensities of these bands were substantially weakened for $\mathrm{SHBC}_{400}$ due to the part decomposition of phenyl groups, and disappeared for the SG film, indicating the completed removal of phenyl groups annealed at $800{ }^{\circ} \mathrm{C}$. Moreover, two peaks at $1384 \mathrm{~cm}^{-1}$ and $1098 \mathrm{~cm}^{-1}$, corresponding to the vibrations of $\mathrm{C}-\mathrm{C}$ bonds and collective ring breathing mode of the aromatic $\mathrm{HBC}-\mathrm{S}$ core,${ }^{59}$ respectively, confirmed the intact HBC-S moieties after annealing at 800 ${ }^{\circ} \mathrm{C}$.

The XRD pattern displayed a broad diffraction peak (oo2) at $20.13^{\circ}$ for $\mathrm{SHBC}$ film and $20.76^{\circ}$ for the SHBC 400 film, corresponding to a d-spacing of $4.4 \AA$ and $4.2 \AA$, respecitively. After annealing at $800{ }^{\circ} \mathrm{C}$, the characteristic (ooz) peak shifted to a larger angle of $22.18^{\circ}$ and one noted a decreased d-spacing of $4.0 \AA$ (Figure 2c), suggestive of the enhanced graphitization of SG film upon annealing process. XPS characterization was performed to probe the chemical composition of the SG, SHBC40o, and SHBC films (Figure 2d,e). From the full XPS spectra (Figure $2 \mathrm{~d}$ ), the characteristic peaks of $\mathrm{C}_{1}$ and S2p were observed. The SG film gave a sulfur doping content of 1.5 at $\%$ while the pristine SHBC and SHBC400 contained $\sim 11.6$ at $\%$ and $\sim 7.1$ at $\%$ sulfur moieties, respectively. High-resolution S2p XPS spectra of SG, SHBC40o and SHBC films differ only in the intensity of the $C$-S peaks reflecting a different content of C-S bonds. All S2p XPS spectra can be deconvoluted into two distinct peaks featuring the $\mathrm{S}-\mathrm{C}$ bond at 165.0 and $163.7 \mathrm{eV}$ (Figure 3d), which are identified as

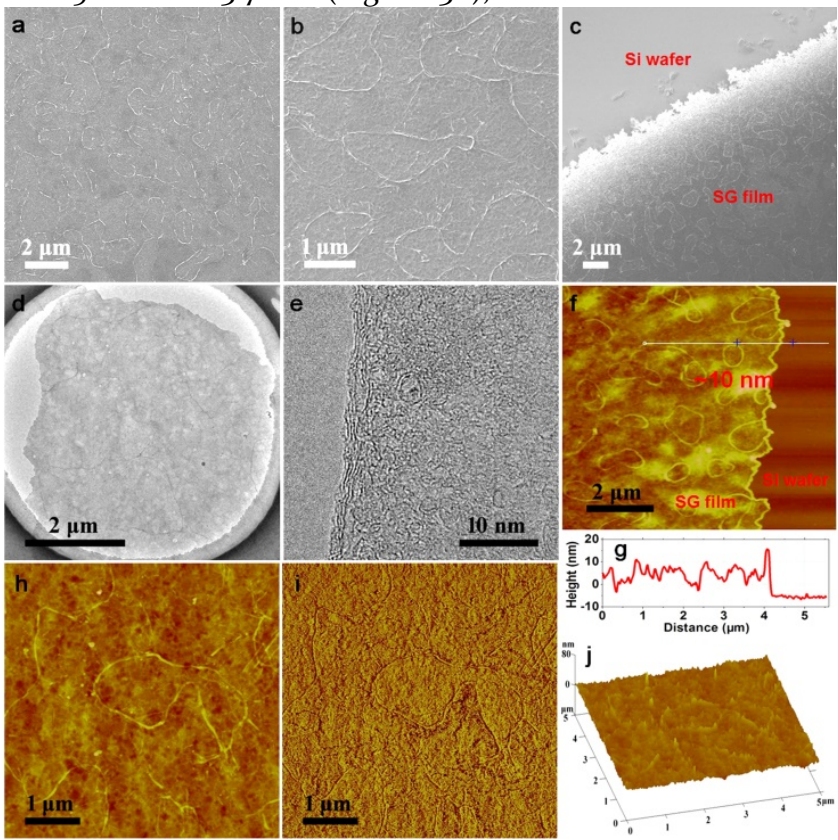

Figure 3. Surface morphology of SHBC-derived SG films. (a) Low-magnification and (b) high-magnification SEM images of SG film. (c) SEM image of the finger edges of the SG film, created by the oxidative etching, showing an edge resolution of around $2 \mu \mathrm{m}$. (d) TEM and (e) HRTEM images of the SG film. (f) AFM height image and (g) height profile of the edge of the SG film, with an average thickness of $\sim 10 \mathrm{~nm}$. (h-j) AFM (h) height image, (i) phase image and (j) 3D surface plot of the top-view of the SG film. 

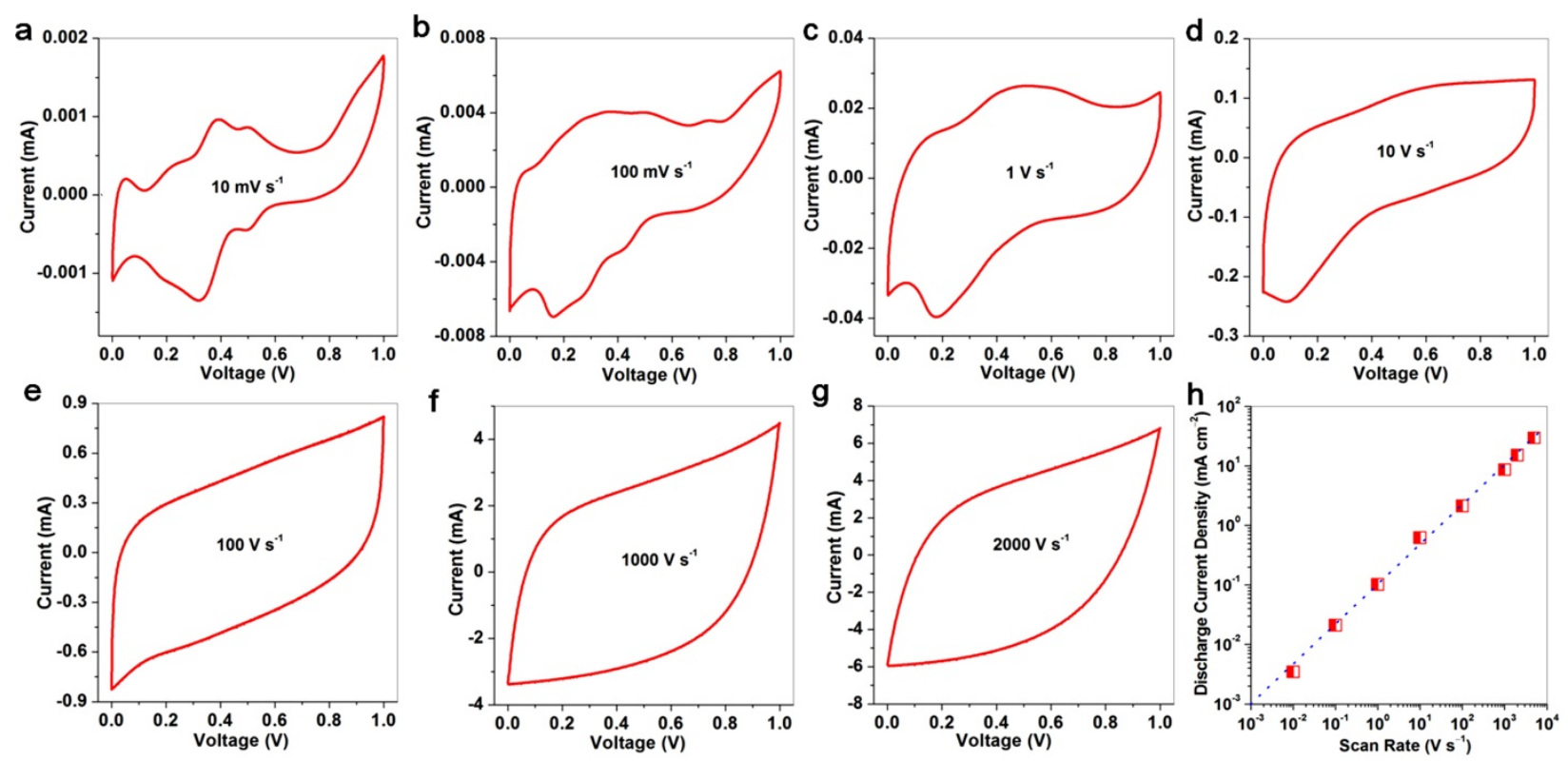

Figure 4. Electrochemical comparison of SG-MSCs. (a-g) CV curves of SG-MSCs measured at different scan rates of (a) o.o1, (b) 0.1, (c) 1, (d) 10, (e) 100, (f) 1000, and (g) $2000 \mathrm{~V} \mathrm{~s}^{-1}$. (h) Plot of the discharge current as a function of the scan rate for SG-MSCs. Linear dependence observed at $2000 \mathrm{Vs}^{-1}$ (blue dot line) suggests ultrahigh power delivery of SG-MSCs.

the spin-orbit coupling positions of $\mathrm{S}_{2} \mathrm{p}_{1 / 2}$ and $\mathrm{S}_{2} \mathrm{p}_{3 / 2}$, respectively. ${ }^{42,47,60}$ For the $\mathrm{SHBC}_{400}$ film, the S2p XPS spectrum exhibited an additional component at $167 \sim 169 \mathrm{eV}$ that was assigned to carbon bonded $\mathrm{SO}_{\mathrm{x}}$ species. ${ }^{46,48}$ This resulted from the decomposition of the SHBC, in which the terminated sulfurs in S-HBC units might interact with oxygen atoms likely adsorbed on samples in air. Further, the bond of C-S $(286.5 \mathrm{eV})$ in the C1s XPS spectrum of the SG film was also unraveled (Figure $\mathrm{S}_{5}$ ). ${ }^{47,60}$ Raman spectra showed the representative broad $D$ and $G$ peaks for $\mathrm{SHBC}_{400}$ and $S G$ film, indicative of the nature of a carbonized film (Figure $\mathbf{2 f}$ ). The decreased $\mathrm{I}_{\mathrm{D}} / \mathrm{I}_{\mathrm{G}}$ ratio from 1.08 for $\mathrm{SHBC}_{400}$ to 0.98 for SG film accounted for an enhanced degree of graphitization, ${ }^{61}$ which is good agreement with XRD result (Figure 2c). In addition, both SHBC and SHBC400 films had an extremely low value of $<0.001 \mathrm{~S} \mathrm{~cm}^{-1}$. Note that the low conductivity of SHBC400 film is ascribe to the partly decomposition of SHBC at $400{ }^{\circ} \mathrm{C}$, consisting of the decomposed phenyl groups and intact HBC-S moieties. By contrast, the asproduced SG film exhibited high electrical conductivity of $\sim 95 \mathrm{~S}$ $\mathrm{cm}^{-1}$, measured by a standard four-point probe system. These results demonstrated the successful fabrication of an Sdoped, conductive SG film derived from a sulfur-annulated nanographene with assistance of Au confinement layer.

The surface topography of the SHBC-derived SG film was further investigated by scanning electron microscopy (SEM), transmission electron microscopy (TEM) and atomic force microscopy (AFM) measurements (Figure 3, and Figure S6S8). Low-magnification SEM (Figure 3a) and TEM (Figure 3d) images of the as-fabricated SG films were continuous and uniform over a large area. High-magnification SEM image (Figure 3b, c) disclosed the formation of the irregularly circular-shaped grain boundaries (Figure S6 and $\mathrm{S}_{7}$ ). High resolution TEM (HRTEM) image displayed the existence of discontinuous graphitic stacked layers (Figure ze and Figure $S_{7}$ ). The AFM height image and phase images as well as $3 \mathrm{D}$ surface plot confirmed irregularly circular-shaped grain bounda- ries and a relatively uniform flat morphology with a typical thickness of $\sim 10.0 \mathrm{~nm}$ for the SG film (Figure $3 \mathrm{f}-\mathrm{j}$, Figure S8). The energy dispersive X-ray (EDX) spectrum and mapping analysis further demonstrated the uniform doping of sulfur into the SG film (Figure S9), identical to the XPS results (Figure 2d,e). The resulting SG film exhibited a low average surface roughness of $R_{a}<2.5 \mathrm{~nm}$ for the whole measurement area (Figure $3 \mathrm{i}, \mathrm{j}$ ).

Electrochemical Characterization of SG-MSCs. The electrochemical performance of SG-MSCs was first examined by cyclic voltammetry (CV) at scan rates ranging from o.o1 to $2000 \mathrm{~V} \mathrm{~s}^{-1}$ (Figure 4a-g). At low scan rates from 10 to $100 \mathrm{mV}$ $\mathrm{s}^{-1}$, the SG-MSCs showed a pronounced pseudocapacitive effect with three pairs of strong redox peaks (Figure 4a, b), supporting the importance of sulfur-doping in carbon films. With increasing scan rate, the CV curves revealed a gradual transition from the pseudocapacitive to the electric doublelayer capacitive behavior with a nearly rectangular CV shape (Figure 4c-f). Remarkably enough, our SG-MSCs possessed ultrafast charging and discharging capability, and could be operated at ultrahigh scan rates of up to $2000 \mathrm{~V} \mathrm{~s}^{-1}$ (Figure $4 \mathrm{~g}$, Figure S10). which is three orders of magnitude higher than that of conventional supercapacitors, and much higher than those of the reported high-power MSCs (Table $\mathrm{S}_{1}$ ), e.g., onion-like carbon (OLC, $\left.200 \mathrm{~V} \mathrm{~s}^{-1}\right),{ }^{23}$ electrochemically rGO $\left(\right.$ ErGO, $\left.400 \mathrm{~V} \mathrm{~s}^{-1}\right){ }^{62}$ and methane plasma reduced graphene (MPG, $\left.1000 \mathrm{~V} \mathrm{~s}^{-1}\right),{ }^{27}$ and vertically aligned CNTs $\left(1000 \mathrm{~V} \mathrm{~s}^{-1}\right){ }^{6_{3}}$ Furthermore, a linear dependence of the logarithmic discharge current upon scan rate was identified up to $2000 \mathrm{~V} \mathrm{~s}^{-1}$ (Figure $4 \mathrm{~h}$ ), characteristic of ultrahigh instantaneous power.

The evolution of the areal capacitance and volumetric capacitance of SG-MSCs with scan rate is shown in Figure 5a. The areal capacitance and volumetric capacitance of SG film for MSCs recorded at $10 \mathrm{mV} \mathrm{s}^{-1}$ were calculated to be $\sim 553 \mu \mathrm{F}$ $\mathrm{cm}^{-2}$ and $\sim 582 \mathrm{~F} \mathrm{~cm}^{-3}$, respectively, both of which are much higher than those of films of undoped reduced graphene ( $\mathrm{RG}, \sim 208 \mu \mathrm{F} \mathrm{\textrm {cm } ^ { - 2 } , ~} \sim 245 \mathrm{~F} \mathrm{~cm}^{-3}$ ), nitrogen-doped graphene 

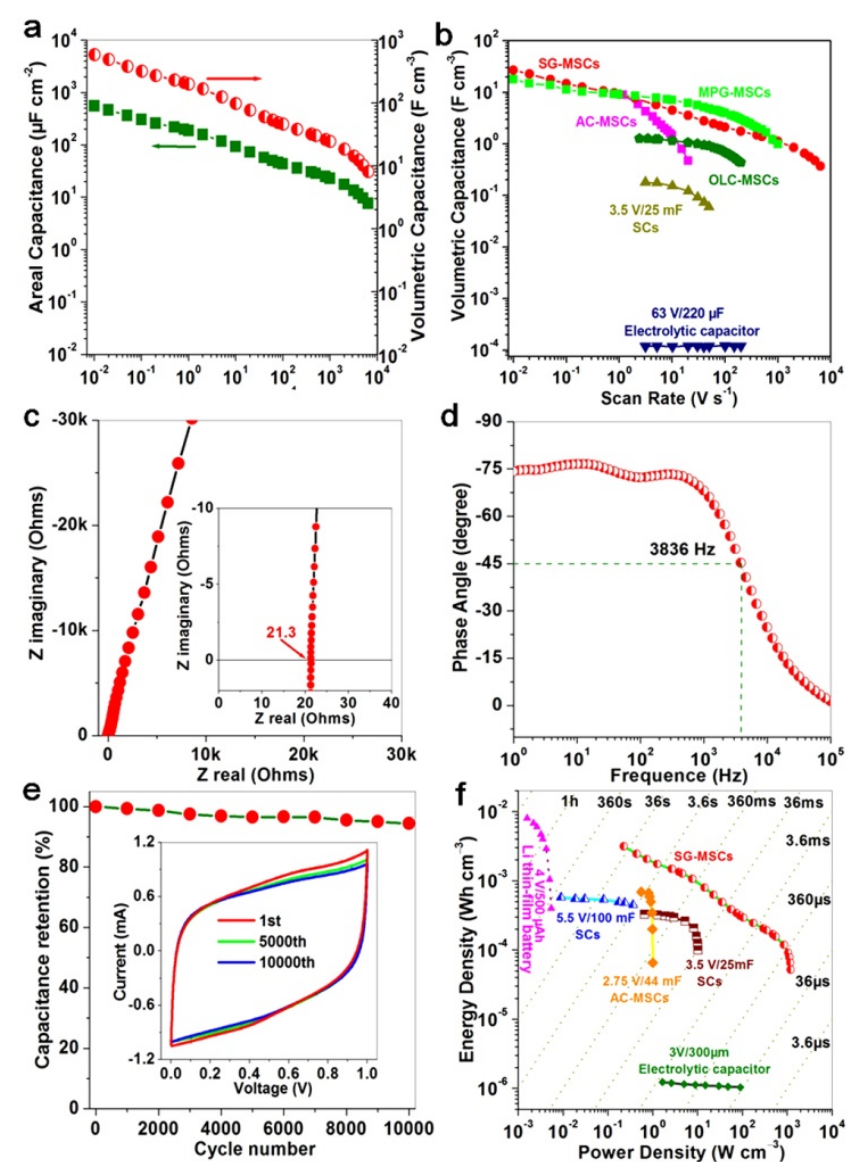

Figure 5. Electrochemical characterization of SG-MSCs. (a) Evolution of areal capacitance (left) and volumetric capacitance versus scan rate of SG film for SG-MSCs (based on single electrode). (b) Comparison of the volumetric capacitance versus scan rate of SG film for SG-MSCs (based on the whole device) with MPG-MSCs, ${ }^{27}$ AC-MSCs, OLC-MSCs, ${ }^{23} 3.5 \mathrm{~V} / 25$ $\mathrm{mF}$ supercapacitors (SCs), and $63 \mathrm{~V} / 220 \mu \mathrm{F}$ electrolytic capacitor. $^{23}$ (c) Complex plane plot of the impedance of SGMSCs. Inset: magnified plot of the high-frequency region. (d) Impedance phase angle as a function of frequency of SGMSCs. The $-45^{\circ}$ phase angle was observed at $3836 \mathrm{~Hz}$, demonstrative of the fast accessibility of the ions in SG-MSCs. (e) Cycling stability of SG-MSCs. Inset: the $1^{\text {st }}, 5000^{\text {th }}$, and $1000{ }^{\text {th }}$ CV curves of SG-MSCs tested at $200 \mathrm{~V} \mathrm{~s}^{-1}$. (f) Ragone plot of SG-MSCs in comparison with the commercially available lithium thin-film batteries $(4 \mathrm{~V} / 500 \mu \mathrm{Ah})$, electrolytic capacitor $(3 \mathrm{~V} / 300 \mu \mathrm{F})$, AC-MSCs $(2.75 \mathrm{~V} / 44 \mathrm{mF})$, supercapacitors (SCs, $3.5 \mathrm{~V} / 25 \mathrm{mF}$, and $5.5 \mathrm{~V} / 100 \mathrm{mF}$ ), suggestive of simultaneously high energy and power densities of SG-MSCs.

(NG, $\sim 361 \mu \mathrm{F} \mathrm{cm} \mathrm{cm}^{-2}, \sim 425 \mathrm{~F} \mathrm{~cm}^{-3}$ ), and boron/nitrogen codoped graphene $\left(\mathrm{BNG}, \sim 415 \mu \mathrm{F} \mathrm{cm}^{-2}, \sim 488 \mathrm{~F} \mathrm{~cm}^{-3}\right.$ ) for MSCs. ${ }^{51}$ It has appeared that heteroatom doping (e.g., $\mathrm{N}, \mathrm{B}, \mathrm{S}$, and $\mathrm{N} / \mathrm{B}$ ) in nanocarbons is highly effective for the enhancement of supercapacitor performance because of the pseudocapacitive effect and the improvement of the interface wettability. ${ }^{33}$ The pronounced enhancement provided by $S$ doping furnishing multiple robust redox peaks in our SG-MSCs has never been disclosed previously. Further, the cell volumetric capacitance of SG-MSCs is well comparable to those of the stateof-the-art MSCs, e.g., MPG-MSCs, ${ }^{27}$ AC-MSCs, ${ }^{23}$ OLCMSCs, ${ }^{23}$ and much higher than that of commercially available supercapacitors $(3.5 \mathrm{~V} / 25 \mathrm{mF})$, and three orders of magnitude higher than that of electrolytic capacitors $(63 \mathrm{~V} / 220 \mu \mathrm{F}),{ }^{23}$ as shown in Fig. 5b. Moreover, our SG-MSCs can still offer a significant cell capacitance of $\sim 8.1 \mathrm{~F} \mathrm{~cm}^{-3}$ even at ultrahigh rate of $2000 \mathrm{~V} \mathrm{~s}^{-1}$ (Fig.5b).

Electrochemical impedance spectroscopy (EIS) of the SGMSCs exhibits a closed $90^{\circ}$ slope without a charge transport semicircle at high frequency (Fig. 5 c), a vertical line intersection with the real axis at low frequency, and a low equivalent series resistance of $21.3 \Omega$ (inset in Fig. $5 \mathrm{C}$ ), suggestive of ultrafast ion diffusion in such a microdevice. ${ }^{64}$ The dependence of the phase angle on the frequency for SG-MSCs (Fig. 5d) furnished a high characteristic frequency $f_{\mathrm{o}}$ of $3836 \mathrm{~Hz}$ at the phase angle of $-45^{\circ}$. Correspondingly, the relaxation time constant $\tau_{o}\left(\tau_{o}=1 / f_{\mathrm{o}}\right)$ was calculated to be only $0.26 \mathrm{~ms}$ for SGMSCs. This value is three orders of magnitude lower than that of conventional AC electrical double layer capacitors (EDLCs, 1 s) ${ }^{64}$ much shorter than those of high-power supercapacitors and MSCs based on liquid-mediated graphene (o.2 0.73 s), ${ }^{65}$ OLC (26 ms), ${ }^{23}$ graphene/PHiooo (1.0 2.9 $\mathrm{ms}){ }^{66}$ sulfuric acid treated $\operatorname{poly}\left(3,4^{-}\right.$ ethylenedioxythiophene):poly(styrenesulfonate) (0.588 $\mathrm{ms}),{ }^{67}$ MPG (0.28 ms), ${ }^{27}$ ErGO (o.17 1 ms), ${ }^{62}$ and vertically oriented graphene $(0.067 \mathrm{~ms}) .{ }^{64}$ In addition, the cycling stability of SG-MSCs was measured for 1000 times at a scan rate of $200 \mathrm{~V} \mathrm{~s}^{-1}$ (Fig.5e), maintaining $\sim 95.0 \%$ of the initial capacitance (Fig.5d).

The Ragone plot in Fig. $5 \mathrm{f}$ compares the volumetric performance of our SG-MSCs with those of commercially available energy-storage devices. ${ }^{68}$ Our microdevice exhibits a vol-

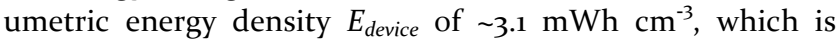
about ten times higher than those of commercially available supercapacitors $\left(3.5 \mathrm{~V} / 25 \mathrm{mF}^{23}\right.$ and $5.5 \mathrm{~V} / 100 \mathrm{mF},<1 \mathrm{mWh}$ $\left.\mathrm{cm}^{-3}\right)$, AC-MSCs $(2.75 \mathrm{~V} / 44 \mathrm{mF})$, and well comparable to lithium thin-film batteries $\left(4 \mathrm{~V} / 500 \mu \mathrm{Ah}, 0.3 \sim 10 \mathrm{mWh} \mathrm{cm}^{-3}\right){ }^{4}$ This energy density value is also higher than those of recently reported thin film MSCs, e.g., MPG $\left(2.5 \mathrm{mWh} \mathrm{cm}^{-3}\right),{ }^{27} \mathrm{NG}$ $\left(2.96 \mathrm{mWh} \mathrm{cm}^{-3}\right),{ }^{51} \mathrm{BNG}\left(3.4 \mathrm{mWh} \mathrm{cm}^{-3}\right),{ }^{51}$ laser scribed graphene $\left(2.0 \mathrm{mWh} \mathrm{cm}^{-3}\right),{ }^{26}$ OLC $\left(1.6 \mathrm{mWh} \mathrm{cm}^{-3}\right),{ }^{23}$ laser written graphene (4.0 $\mathrm{mWh} \mathrm{cm}^{-3}$ ) (Table $\left.\mathrm{S}_{1}\right) .{ }^{25}$ Remarkably enough, our SG-MSCs offered a power density $P_{\text {device }}$ of $\sim 1191 \mathrm{~W} \mathrm{~cm}{ }^{-3}$, which is the highest value reported so far, and comparable to that of high-power electrolytic capacitors $\left(10^{1} \sim 10^{3} \mathrm{~W} \mathrm{~cm}^{-3}\right) .{ }^{69}$

\section{CONCLUSION}

In summary, we have demonstrated the first case of bottom-up, wafer-scale production for highly continuous, uniform, ultrathin SG films derived from a sulfur-decorated nanographene. This fabrication strategy using nanographene molecule as precursor, with assistance of a thin Au layer as $2 \mathrm{D}$ confining cover, provided a new scaffold for constructing large-area carbon films with uniform $\mathrm{S}$ doping, high conductivity and expanded interlayer spacing for high-performance MSCs. The films showed an unprecedented volumetric capacitance of $\sim 582 \mathrm{~F} \mathrm{~cm}^{-3}$, remarkable scan rate of $2000 \mathrm{~V} \mathrm{~s}^{-1}$, ultrafast frequency response with a time constant of $0.26 \mathrm{~ms}$,

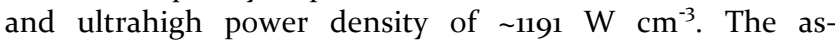
produced SG film will offer numerous opportunities as an outstanding carbon-based material for electrochemical energy storage and conversion systems, such as metal-free oxygen reduction catalysts, ${ }^{47,}{ }^{61} \mathrm{Li}-\mathrm{S}$ batteries, and sensors.

\section{EXPERIMENTAL SECTION}


Preparation of SG films. The precursor of SHBC was first synthesized by thiolation of perchlorinated $\mathrm{HBC}$, as described in our previous work. ${ }^{53,54}$ Then, a stable SHBC dispersion of $0.5 \mathrm{mg} / \mathrm{mL}$ in dichloromethane was dissolved by sonication for $10 \mathrm{~min}$, and subsequently spin-coated (2000 rpm, 60 s; Headway Research Inc.) on the oxygen plasma-treated silicon wafer (single size polished, $300 \mathrm{~nm} \mathrm{SiO}$ layer, Si-Mat) using $300 \mathrm{~W}$ rf power for $10 \mathrm{~min}$ (Plasma System 200-G, Technics Plamsa $\mathrm{GmbH}$ ), and the spin-coating steps were repeated several times until the desirable SHBC film thickness was achieved. Afterwards, $40 \mathrm{~nm}$ Au protecting layer (Premion, 99.9985\% metals basis, Alfa Aesar) was thermally evaporated (EDWARDS FL40o) on the SHBC film with a rate of $\sim 1.0 \AA \mathrm{s}^{-1}$ and chamber pressure of $\sim 2 \times 10^{-6}$ Torr. With assistance of Au layer, the SHBC films were thermally annealed first at $400{ }^{\circ} \mathrm{C}$ for $30 \mathrm{~min}$, and then $800{ }^{\circ} \mathrm{C}$ for $30 \mathrm{~min}$, with a heating rate of $\sim 5^{\circ} \mathrm{C} / \mathrm{min}$, by an AIXTRON furnace (Nanoinstruments Black Magic) in argon gas of $100 \mathrm{sccm}$. After the removal of Au layer etching by a $\mathrm{KI} / \mathrm{I}_{2}$ aqueous solution, the SG films were readily produced for further characterization and device manufacturing.

Fabrication of SG-MSCs. To fabricate the SG-MSCs, 30 nm gold was first thermally evaporated (EDWARDS FL40o) on the SG film with a rate of $\sim 1.0 \AA \mathrm{s}^{-1}$ and chamber pressure of $\sim 2 \times 10^{-6}$ Torr through a home-made 30-interdigitated finger mask (widths of $210 \mu \mathrm{m}$, interspaces of $70 \mu \mathrm{m}$ ). After that, the electrode micro-patterns of SG film on Si wafer were created by oxidative etching of the exposed SG film in an $\mathrm{O}_{2}$ plasma cleaner with $20 \mathrm{sccm} \mathrm{O}_{2}$ flow for $90 \sim 150 \mathrm{~s}$ and $200 \mathrm{~W}$ rf power (Plasma System 200-G, Technics Plamsa GmbH). Afterwards, $\mathrm{H}_{2} \mathrm{SO}_{4} / \mathrm{PVA}$ gel electrolyte was slowly dropcasted onto the surface of interdigitated fingers and solidified overnight. Finally, the all-solid-state planar SG-MSCs were obtained. The gel electrolyte was prepared by mixing 3 g PVA and $3 \mathrm{~g} \mathrm{H}_{2} \mathrm{SO}_{4}$ into $30 \mathrm{ml}$ DI water, and heated at $8 \mathrm{o}^{\circ} \mathrm{C}$ for $1 \mathrm{~h}$ under stirring.

Characterization. Materials characterization were carried out by SEM (Gemini 1530 LEO), optical microscopy, AFM (Veeco Dimension 310o), surface profiler (KLA Tencor P-16+), XRD (SEIFERT XRD 3000 TT Bragg-Brentano diffractometer with $\mathrm{Cu} \mathrm{Ka}$ radiation between $10^{\circ}$ and $60^{\circ}$ and an incident wavelength of $0.15418 \mathrm{~nm}$ ), FT-IR (Nicolet iS 50 ), TGA (STA $449 \mathrm{~F}_{3}$, from 25 to $800{ }^{\circ} \mathrm{C}$ with a heating rate of $5 \%$ min in nitrogen gas), Raman spectra (Bruker, $532 \mathrm{~nm}$ ) and XPS (Omicron Multiprobe equipped with the monochromatic $\mathrm{Al}$ $\mathrm{K}_{\mathrm{a}}$ source, electron analyzer resolution of $0.9 \mathrm{eV}$ ). For TEM characterization, the SG film was delaminated from the $\mathrm{SiO}_{2} / \mathrm{Si}$ wafer in the HF solution $(40 \%)$, and rinsed with deionized water three times. After that, the delaiminated SG film was carefully transferred into $\mathrm{Cu}$ grids. The electrical conductivity of the SG film was measured by a standard fourpoint probe system with a Kiethley 2700 Multimeter. The electrochemical properties was examined by $\mathrm{CV}$ at the scan rates of $0.01 \sim 5000 \mathrm{~V} \mathrm{~s}^{-1}$, galvanostatic charge and discharge profiles at different current density, and EIS recorded in the frequency range of $1 \sim 100 \mathrm{kHz}$ with a $5 \mathrm{mV}$ ac amplitude, using a $\mathrm{CHI} 760 \mathrm{D}$ electrochemical workstation.

\section{ASSOCIATED CONTENT}

This material is available free of charge via the Internet at http://pubs.acs.org.
Calculation of areal/volumetric capacitance, power/energy density, TEM, SEM, AFM and EDX analysis characterization of SG film, CVs of SG-MSCs, and Table Si. (PDF)

\section{AUTHOR INFORMATION}

\section{Corresponding Author}

*wuzs@dicp.ac.cn;

*xinliang.feng@tu-dresden.de;

*muellen@mpip-mainz.mpg.de

\section{Notes}

The authors declare no competing financial interests.

\section{ACKNOWLEDGMENT}

This work was financially supported by the National Natural Science Foundation of China (Grant 51572259), Ministry of Science and Technology of China (Grant 2016YBFo10o1oo and 2016YFA0200200), Natural Science Foundation of Liaoning Province (Grant 201602737), Thousand Youth Talents Plan of China, DICP (Grant Y5610121T3), China Postdoctoral Science Foundation (Grant 2016M601349), dedicated funds for methanol conversion from DICP, ERC Grant on 2DMATER, and EC under Graphene Flagship (No. CNECT-ICT-6o4391).

\section{REFERENCES}

(1) LeMieux, M. C.; Bao, Z. N. Nat. Nanotechnol. 20o8, 3, 585.

(2) Gates, B. D. Science 2oo9, 323, 1566.

(3) Liu, C.; Li, F.; Ma, L. P.; Cheng, H. M. Adv. Mater. 2010, 22 , E28.

(4) Yu, D. S.; Goh, K.; Wang, H.; Wei, L.; Jiang, W. C.; Zhang, Q.; Dai, L. M.; Chen, Y. Nat. Nanotechnol. 2014, 9, 555.

(5) Wang, X.; Lu, X.; Liu, B.; Chen, D.; Tong, Y.; Shen, G. Adv. Mater. 2014, 26, 4763.

(6) Wen, L.; Li, F.; Cheng, H.-M. Adv. Mater. 2016, 28, 4306.

(7) Wan, S.; Peng, J.; Jiang, L.; Cheng, Q. Adv. Mater. 2016, 28, 7862.

(8) Chmiola, J.; Largeot, C.; Taberna, P. L.; Simon, P.; Gogotsi, Y. Science 2010, 328, 480 .

(9) Huang, P.; Lethien, C.; Pinaud, S.; Brousse, K.; Laloo, R.; Turq, V.; Respaud, M.; Demortière, A.; Daffos, B.; Taberna, P. L.; Chaudret, B.; Gogotsi, Y.; Simon, P. Science 2016, 351, 691.

(10) Beidaghi, M.; Gogotsi, Y. Energy Environ. Sci. 2014, 7, 867.

(11) Wu, Z.-S.; Feng, X.; Cheng, H.-M. Natl Sci. Rev. 2014, 1, 277.

(12) Wang, S.; Zheng, S. H.; Wu, Z.-S.; Sun, C. L. Sci. Sin. Chim. 2016, 46,1 .

(13) Ferris, A.; Garbarino, S.; Guay, D.; Pech, D. Adv. Mater. 2015 27, 6625 .

(14) Su, Z.; Yang, C.; Xie, B.; Lin, Z.; Zhang, Z.; Liu, J.; Li, B.; Kang, F.; Wong, C. P. Energy Environ. Sci. 2014, 7, 2652.

(15) Kurra, N.; Alhebshi, N. A.; Alshareef, H. N. Adv. Energy Mater. 2014, 4, 1401303.

(16) Zhu, Y. G.; Wang, Y.; Shi, Y.; Wong, J. I.; Yang, H. Y. Nano Energy 2014, 3, 46.

(17) Wang, K.; Zou, W. J.; Quan, B. G.; Yu, A. F.; Wu, H. P.; Jiang, P.; Wei, Z. X. Adv. Energy Mater. 2011, 1, 1068.

(18) Liu, S.; Gordiichuk, P.; Wu, Z.-S.; Liu, Z.; Wei, W.; Wagner, M.; Mohamed-Noriega, N.; Wu, D.; Mai, Y.; Herrmann, A.; Müllen, K.; Feng, X. Nat. Commun. 2015, 6, 8817.

(19) Zhai, Y.; Dou, Y.; Zhao, D.; Fulvio, P. F.; Mayes, R. T.; Dai, S. Adv. Mater. 2011, 23, 4828.

(20) Kurra, N.; Ahmed, B.; Gogotsi, Y.; Alshareef, H. N. Adv. Energy Mater. 2016, 1601372.

(21) Wei, L.; Nitta, N.; Yushin, G. ACS Nano 2o13, 7, 6498. 
(22) Huang, H.-C.; Chung, C.-J.; Hsieh, C.-T.; Kuo, P.-L.; Teng, H. Nano Energy 2016, 21, 90.

(23) Pech, D.; Brunet, M.; Durou, H.; Huang, P. H.; Mochalin, V.; Gogotsi, Y.; Taberna, P. L.; Simon, P. Nat. Nanotechnol. 2010, 5, 651.

(24) Kim, S. K.; Koo, H. J.; Lee, A.; Braun, P. V. Adv. Mater. 2014, 26, 5108.

(25) Gao, W.; Singh, N.; Song, L.; Liu, Z.; Reddy, A. L. M.; Ci, L. J.; Vajtai, R.; Zhang, Q.; Wei, B. Q.; Ajayan, P. M. Nat. Nanotechnol. 2011, 6, 496.

(26) El-Kady, M. F.; Kaner, R. B. Nat. Commun. 2013, 4, 1475.

(27) Wu, Z.-S.; Parvez, K.; Feng, X. L.; Müllen, K. Nat. Commun. 2013, 4, 2487.

(28) Liu, Z.; Wu, Z.-S.; Yang, S.; Dong, R.; Feng, X.; Müllen, K. Adv. Mater. 2016, 28, 2217.

(29) Niu, Z. Q.; Zhang, L.; Liu, L. L.; Zhu, B. W.; Dong, H. B.; Chen, X. D. Adv. Mater. 2013, 25, 4035.

(3о) Wu, Z.-K.; Lin, Z.; Li, L.; Song, B.; Moon, K.-s.; Bai, S.-L.; Wong, C.-P. Nano Energy 2014, 10, 222.

(31) Jeong, H. M.; Lee, J. W.; Shin, W. H.; Choi, Y. J.; Shin, H. J.; Kang, J. K.; Choi, J. W. Nano Lett. 2011, 11, 2472.

(32) Wen, Z.; Wang, X.; Mao, S.; Bo, Z.; Kim, H.; Cui, S.; Lu, G.; Feng, X.; Chen, J. Adv. Mater. 2012, 24, 5610.

(33) Lin, T.; Chen, I.-W.; Liu, F.; Yang, C.; Bi, H.; Xu, F.; Huang, F. Science 2015, 350, 1508.

(34) Yang, L. J.; Jiang, S. J.; Zhao, Y.; Zhu, L.; Chen, S.; Wang, X. Z.; Wu, Q.; Ma, J.; Ma, Y. W.; Hu, Z. Angew. Chem. Int. Ed. 2o11, 50, 7132 .

(35) Wang, D. W.; Li, F.; Chen, Z. G.; Lu, G. Q.; Cheng, H. M. Chem. Mater. 2008, 20, 7195.

(36) Wu, Z.-S.; Ren, W. C.; Xu, L.; Li, F.; Cheng, H. M. ACS Nano 2011, 5, 5463.

(37) Wen, Y. Y.; Wang, B.; Huang, C. C.; Wang, L. Z.; HulicovaJurcakova, D. Chem.-Eur. J. 2015, 21, 80.

(38) Hulicova-Jurcakova, D.; Puziy, A. M.; Poddubnaya, O. I.; Suarez-Garcia, F.; Tascon, J. M. D.; Lu, G. Q. J. Am. Chem. Soc. 2009, 131, 5026.

(39) Huang, C. C.; Puziy, A. M.; Sun, T.; Poddubnaya, O. I.; Suarez-Garcia, F.; Tascon, J. M. D.; Hulicova-Jurcakova, D. Electrochim. Acta 2014, 137, 219.

(40) Deng, W. F.; Zhang, Y. J.; Yang, L.; Tan, Y. M.; Ma, M.; Xie, Q. J. RSC Adv. 2015, 5, 13046.

(41) Zhang, J. L.; Jiang, M.; Xing, L. B.; Qin, K.; Liu, T. Z.; Zhou, J.; Si, W. J.; Cui, H. Y.; Zhuo, S. P. Chin. J. Chem. 2016, 34, 46.

(42) Chen, X.; Chen, X. H.; Xu, X.; Yang, Z.; Liu, Z.; Zhang, L. J.; $\mathrm{Xu}, \mathrm{X}$. J.; Chen, Y.; Huang, S. M. Nanoscale 2014, 6, 13740.

(43) Hulicova-Jurcakova, D.; Seredych, M.; Lu, G. Q.; Bandosz, T. J. Adv. Funct. Mater. 2009, 19, 438.

(44) Ai, W.; Luo, Z.; Jiang, J.; Zhu, J.; Du, Z.; Fan, Z.; Xie, L.; Zhang, H.; Huang, W.; Yu, T. Adv. Mater. 2014, 26, 6186.

(45) Wu, Z.-S.; Winter, A.; Chen, L.; Sun, Y.; Turchanin, A.; Feng, X. L.; Müllen, K. Adv. Mater. 2012, 24, 5130.

(46) Jeon, I.-Y.; Zhang, S.; Zhang, L.; Choi, H.-J.; Seo, J.-M.; Xia, Z.; Dai, L.; Baek, J.-B. Adv. Mater. 2013, 25, 6138.
(47) Yang, Z.; Yao, Z.; Li, G. F.; Fang, G. Y.; Nie, H. G.; Liu, Z.; Zhou, X. M.; Chen, X.; Huang, S. M. ACS Nano 2012, 6, 205.

(48) Higgins, D.; Hoque, M. A.; Seo, M. H.; Wang, R. Y.; Hassan, F.; Choi, J. Y.; Pritzker, M.; Yu, A. P.; Zhang, J. J.; Chen, Z. W. Adv. Funct. Mater. 2014, 24, 4325.

(49) Zhi, L. J.; Müllen, K. J. Mater. Chem. 2oo8, 18, 1472.

(50) Li, X.; Song, Q.; Hao, L.; Zhi, L. Small 2014, 10, 2122.

(51) Wu, Z.-S.; Parvez, K.; Winter, A.; Vieker, H.; Liu, X.; Han, S.; Turchanin, A.; Feng, X.; Müllen, K. Adv. Mater. 2014, 26, 4552.

(52) Peng, Z. W.; Ye, R. Q.; Mann, J. A.; Zakhidov, D.; Li, Y. L.; Smalley, P. R.; Lin, J.; Tour, J. M. ACS Nano 2015, 9, 5868.

(53) Tan, Y. Z.; Osella, S.; Liu, Y.; Yang, B.; Beljonne, D.; Feng, X. L.; Mullen, K. Angew. Chem. -Int. Ed. 2015, 54, 2927.

(54) Tan, Y. Z.; Yang, B.; Parvez, K.; Narita, A.; Osella, S.; Beljonne, D.; Feng, X.; Müllen, K. Nat. Commun. 2o13, 4, 2646.

(55) Vance, A. L.; Willey, T. M.; Nelson, A. J.; van Buuren, T.; Bostedt, C.; Terminello, L. J.; Fox, G. A.; Engelhard, M.; Baer, D. Langmuir 2002, 18, 8123 .

(56) Yao, Y. X.; Fu, Q.; Zhang, Y. Y.; Weng, X. F.; Li, H.; Chen, M. S.; Jin, L.; Dong, A. Y.; Mu, R. T.; Jiang, P.; Liu, L.; Bluhm, H.; Liu, Z.; Zhang, S. B.; Bao, X. H. Proc. Natl. Acad. Sci. U S A 2014, 111, 17023 .

(57) Deng, D.; Novoselov, K. S.; Fu, Q.; Zheng, N.; Tian, Z.; Bao, X. Nat. Nanotechnol. 2016, 11, 218.

(58) Wang, P.-I.; Pisula, W.; Mullen, K.; Liaw, D.-J. Polym. Chem. 2016, $7,6211$.

(59) Maghsoumi, A.; Narita, A.; Dong, R. H.; Feng, X. L.; Castiglioni, C.; Mullen, K.; Tommasini, M. Phys. Chem. Chem. Phys. 2016, 18, 11869.

(6o) Tucek, J.; Blonski, P.; Sofer, Z.; Simek, P.; Petr, M.; Pumera, M.; Otyepka, M.; Zboril, R. Adv.Mater. 2o16, 28, 5045.

(61) Ma, Z.; Dou, S.; Shen, A.; Tao, L.; Dai, L.; Wang, S. Angew. Chem. -Int. Ed. 2015, 54, 1888.

(62) Sheng, K. X.; Sun, Y. Q.; Li, C.; Yuan, W. J.; Shi, G. Q. Sci. Rep. 2012, 2, 247.

(63) Ghosh, A.; Le, V. T.; Bae, J. J.; Lee, Y. H. Sci. Rep. 2013, 3, 2939.

(64) Miller, J. R.; Outlaw, R. A.; Holloway, B. C. Science 2010 , 329, 1637 .

(65) Yang, X. W.; Cheng, C.; Wang, Y. F.; Qiu, L.; Li, D. Science 2013, 341, 534 .

(66) Wu, Z.-S.; Liu, Z.; Parvez, K.; Feng, X.; Müllen, K. Adv. Mater. 2015, 27, 3669.

(67) Zhang, M.; Zhou, Q. Q.; Chen, J.; Yu, X. W.; Huang, L.; Li, Y. R.; Li, C.; Shi, G. Q. Energy Environ. Sci. 2016, 9, 2005.

(68) Gogotsi, Y.; Simon, P. Science 2012, 335, 167.

(69) El-Kady, M. F.; Strong, V.; Dubin, S.; Kaner, R. B. Science 2012, 335, 1326. 


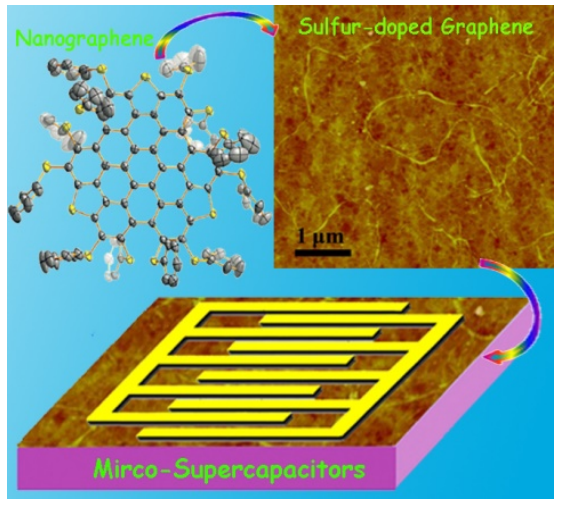

